\section{First Benedicks Effect in Gas-free Mercury, as Influenced by the Mean Temperature}

IN a previous letter ${ }^{1}$ we have shown that, in mercury carefully freed from gas ions, the First Benedicks effect exists with a positive sign, whereas in the presence of gas ions the effect had shown a negative sign. The influence of gas ions actually seems thoroughly to modify the thermoelectric properties of some metals.

In view of the fundamental character of this influence, the experiments on mercury have now been repeated, using a strangulation apparatus made of silica, with a new heating arrangement (hot and cold flowing oil), and also a photographic registration method. Fig. 1 exemplifies the registrations finally obtained : in the upper parts are to be seen the relayamplified deflections of the galvanometer, showing small oscillations due to Brownian movement; below, the corresponding tem. perature registrations.

The new observations were found entirely to confirm the positive character of the effect in gas-free mercury. They further reveal a new, important fact.

From a quantitative point of view, the effect is determined by the formula

$$
u=K \cdot \frac{3}{\triangle t}
$$

The parameter $K$. depending on the metal and, to a certain extent, on the apparatus used (here No. 20), and hence now designated as $K_{20}$, has been calculated from the observations, using (1), and plotted as ordinates in Fig. 2, where abscissæe are the mean temperatures $t_{\text {med }}$.

It will be seen that the effect varies with the temperature, increasing in a strongly accelerated way.

It is interesting that this increase, with increasing temperature, of the First Benedicks effect appears to be just the same as that which has been discovered earlier for the reversed effect, the Second Benedicks

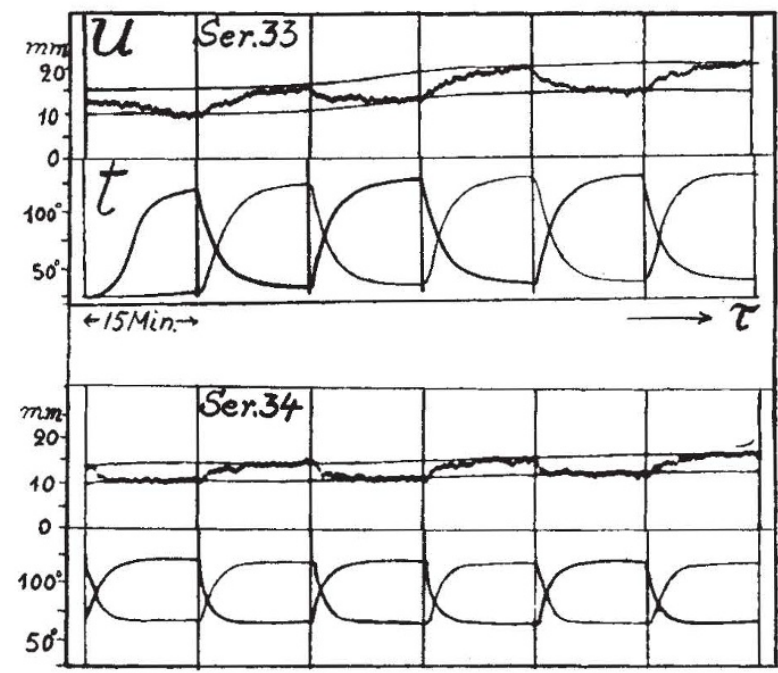

Fig. I.

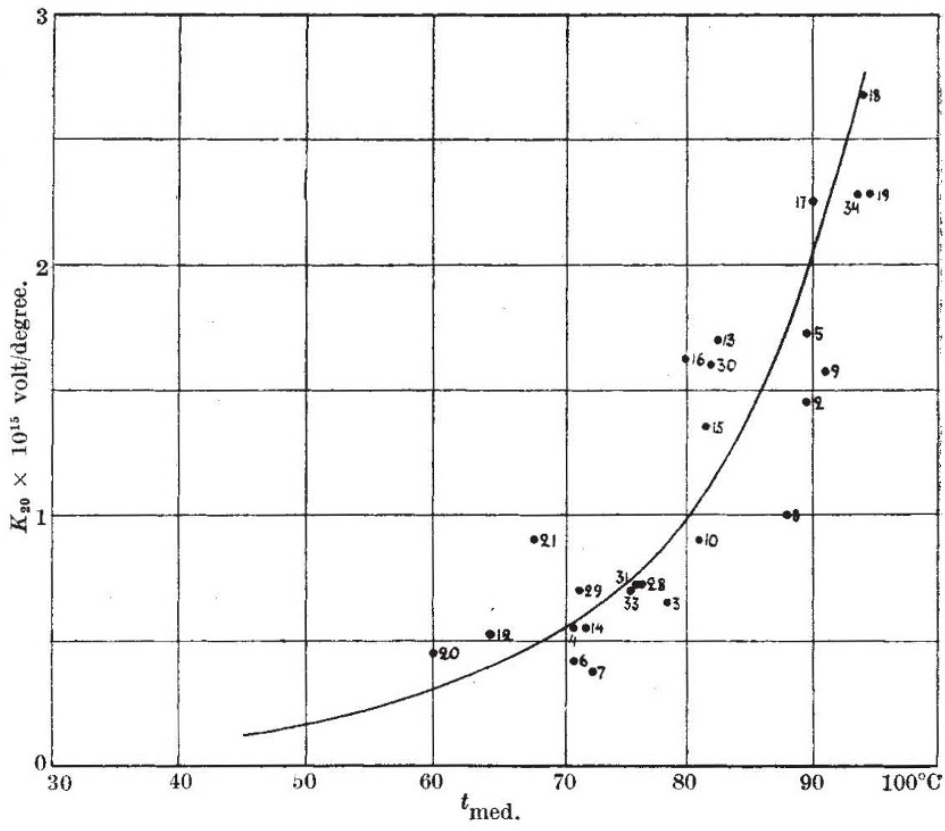

Fig. 2.

First Benedicks effect in mercury.

effect ${ }^{2}$, and which presents a striking similarity to the well-known Richardson formula, valid for electron emission from glowing metals. It actually appears plausible that the emission of electrons along a steep temperature gradient in the metal may follow the same law as the emission into vacuum.

$$
\text { C. Benedicks. }
$$

Metallografiska Institutet,

\section{P. Sederholm.} Stockholm.

${ }^{1}$ Nature, 141, 1097 (1938).

${ }^{2}$ Benedicks, C., and Siljeholm, G., Ark. Mat. Astron. Fys., Stockholm, 24 A, No. 7 (1933), (see Fig. 41)

\section{Magnetic Anisotropy of Diphenyldiacetylene}

E. H. WIEBenga has recently determined the crystal structure of diphenyldiacetylene ${ }^{1}$, $\left(\mathrm{C}_{6} \mathrm{H}_{5}\right)$.C:C.C:C. $\left(\mathrm{C}_{6} \mathrm{H}_{5}\right)$. The molecule is plane, with a straight carbon chain, the length of the molecule making angles of approximately $48^{\circ}, 52^{\circ}, 78^{\circ}$ with the $a, b, c$ axes of the monoclinic unit cell. (By inadvertence, the complements of the first two angles were given in Dr. Wiebenga's letter in Nature. He has since very kindly sent me the exact direction cosines determined by means of a Fourier analysis, and has also provided me with some beautiful crystals for this investigation.)

The magnetic anisotropy of the crystals, weighing about $4 \mathrm{mgm}$. each, density $1 \cdot 170 \mathrm{gm}$./c.c. at $20^{\circ} \mathrm{C}$., was determined by Krishnan's second method in a field of some 7,000 oersted. The minimum diamagnetic susceptibility was determined by the flotation method. The principal crystal susceptibilities are $\chi_{1}=-93.8 \times 10^{-6}( \pm 0.5), \chi_{2}=-188 \cdot 0 \times 10^{-6}$ $\chi_{3}=-109 \cdot 6 \times 10^{-6}, \psi=-88 \cdot 5^{\circ}$. Hence the mean 